\title{
The LHCb upgrade
}

\author{
Umberto Marconi* \\ INFN Sezione di Bologna \\ E-mail: umberto.marconi@bo.infn.it
}

During the LHC Run 1 LHCb has successfully performed a very large number of world-class precision measurements in heavy flavour physics by collecting over $3 \mathrm{fb}^{-1}$ at centre-of-mass energies of $7 \mathrm{TeV}$ and $8 \mathrm{TeV}$. However, even after an additional expected integrated luminosity of $5-6 \mathrm{fb}^{-1}$ in Run 2, many of the LHCb measurements will remain limited by statistics. The current $1 \mathrm{MHz}$ readout system is the main bottle neck to run $\mathrm{LHCb}$ at higher luminosity and with higher trigger efficiencies. LHCb will therefore undergo a major upgrade in LS 2 (2018 - 2019) aimed at collecting an order of magnitude more data by 2028 . The upgrade consists of a new full readout at the LHC bunch crossing rate $(40 \mathrm{MHz})$ with the ultimate flexibility of only a software trigger. In order to increase the instantaneous luminosity up to $2 \times 10^{33} \mathrm{~cm}^{-2} \mathrm{~s}^{-1}$, several subdetector upgrades are also underway to cope with the higher occupancies and radiation dose.

INFN Workshop on Future Detectors for HL-LHC,

March 11-13, 2014

Trento, Italy

* Speaker. 


\section{Introduction}

The main objective of LHCb experiment [1] at the CERN Large Hadron Collider (LHC) is measuring indirect effects of New Physics (NP) beyond the Standard Model (BSM). The charm and beauty quark sectors offer a large variety of decay modes and topologies to explore in search of NP. Of particular interest are the rare decay modes that are strongly suppressed in the Standard Model, involving loop and penguin diagrams, in Flavour Changing Neutral Current interactions. $\mathrm{LHCb}$ performs high precision measurements, accessing as many decay modes as possible.

The understanding of flavour dynamics is one of the most important aims of particle physics. The last 15 years have witnessed the triumph of the Kobayashi-Maskawa (KM) mechanism, formalised in the Cabibbo-Kobayashi-Maskawa (CKM) quark mixing matrix, which describes all flavour changing transitions of quarks in the SM. This has been achieved owing to several experiments, notably including those operating at the so called B-factories (BaBar and Belle), at the Tevatron (CDF and D0), and now at the LHC (ATLAS, CMS and LHCb). LHCb has produced a plethora of results, on a broad range of flavour observables, and ATLAS and CMS have given significant contributions to the beauty sector, mainly using final states containing muon pairs. The measurements performed confirm that the level of $\mathrm{CP}$ violation in the SM is not large enough to explain the matter-antimatter asymmetry of the universe. This is indeed considered as a compelling evidence that other sources of CP violation BSM must exist.

LHCb can profit from a number of powerful key features. The $b \bar{b}$ production cross-section is expected to be around $500 \mu \mathrm{b}$ at $\sqrt{s}=14 \mathrm{TeV}$, implying a rate of about $200 \mathrm{kHz}$ of b-events at the LHCb interaction point, running at the instantaneous luminosity of $4 \times 10^{32} \mathrm{~cm}^{-2} \mathrm{~s}^{-1}$. The production mechanism at the LHC gives access to all the b-flavoured hadrons. The cross-sections for $c \bar{c}$ production is approximately 20 times larger than $b \bar{b}$. The hadronic states containing quarks of the $b \bar{b}$ and $c \bar{c}$ pairs appear in the same hemisphere and with a large boost. This allows a very good proper time resolution (for resolving, for instance, the fast $B_{s}$ oscillations), and it also allows the flavour tagging with a single arm spectrometer.

The LHCb detector acceptance spans the polar angles between $15 \mathrm{mrad}$ to $300 \mathrm{mrad}$ in the horizontal bending plane of the spectrometer magnet and $250 \mathrm{mrad}$ in the vertical non-bending plane, equivalent to a pseudo-rapidity range $2<\eta<5$. Although the acceptance corresponds to $4 \%$ of the solid angle, it includes about $40 \%$ of the $b \bar{b}$ pair production cross-section. LHCb has a full detector coverage in the complete pseudo-rapidity range, including tracking, vertex reconstruction, particle identification and calorimetry.

The current detector and the trigger have been operated efficiently at four times the design pileup. In addition, the physics output rate was increased from $2 \mathrm{kHz}$ in 2010 to $5 \mathrm{kHz}$ in 2012, to satisfy an extended physics program. In these conditions the LHCb sub-detectors have performed equivalently or even better than design. The VELO vertex detector has achieved an impact parameter resolution of about $20 \mu \mathrm{m}$, for tracks with high transverse momenta (below $10 \mathrm{GeV} / \mathrm{c}$ in the LHCb acceptance). The proper time resolution measured for multi-body decays as $B_{s} \rightarrow J / \psi \phi$ and $B_{s} \rightarrow D_{s} \pi$ is around $45 \mathrm{fs}$. The momentum resolution ranges from $0.4 \%$ at $5 \mathrm{GeV} / \mathrm{c}$, up to $0.6 \%$ at $100 \mathrm{GeV} / \mathrm{c}$. The mass resolution achieved for the $J / \psi \rightarrow \mu \mu$ decays is of about $15 \mathrm{MeV} / \mathrm{c}^{2}$ and it is of $8 \mathrm{MeV} / \mathrm{c}^{2}$ for the $B_{S} \rightarrow J / \psi \phi$ decays, using the mass constraints. The kaon identification is $95 \%$ with only $5 \%$ misidentification of pions. Muons are identified with $97 \%$ efficiency with 
only $1-3 \%$ of mis-identification. The energy resolution of the electromagnetic calorimeter has a sampling term less than $10 \%$ with a $1 \%$ constant term.

The present LHCb trigger is organised in two levels. The first level consists of a low-latency hardware trigger, operating in a few $\mu$ s. It uses simple events signatures, as transverse energy and momentum of electrons, photons, hadrons and muons, to reduce the readout rate to about $1 \mathrm{MHz}$. The LHCb visible event rate of $12 \mathrm{MHz}$ is reduced to $1 \mathrm{MHz}$ by thresholds of $1 \mathrm{GeV} / \mathrm{c}$ for muons and $3-4 \mathrm{GeV} / \mathrm{c}$ for electrons, photons and hadrons. The available bandwidth to the HLT is shared between $150 \mathrm{kHz}$ of electron and photon triggers, $450 \mathrm{kHz}$ of hadron triggers and $400 \mathrm{kHz}$ of muon triggers. The HLT has access to the full detector information and performs exclusive and inclusive event selections by means of 30,000 copies of the trigger algorithm running on 1,600 CPU nodes. Operating at $\sqrt{s}=8 \mathrm{TeV}$, with an output rate of $5 \mathrm{kHz}$, the combined trigger efficiency is of $90 \%$ for selecting $B$ decays to muons, $30 \%$ for selecting $B$ decays to hadrons, and $10 \%$ for charm decays (efficiencies are referred to events that will be useful to the offline analysis).

LHCb has remarkably pioneered several operational developments during Run 1, aiming at maximising the physics yield and reducing the systematics. A real-time control system has been put in place that allows LHCb to run at a constant and optimised luminosity throughout the fills. It implies that a single trigger configuration and pre-scales settings can be used permanently. It leads to stable detector performance over time and easier calibrations, which in itself reduces systematics. LHCb invented a new concept to increase the High Level Trigger (HLT) CPU capacity by more than $20 \%$. It has been achieved by deferring the events accepted at the first level trigger to local disks in the HLT farm, to then process them through the HLT during the inter-fill time. The physics results of Run 1 relies on these features, that will be exploited also in the future.

In addition to the routine operation LHCb has taken many occasions to explore the detector response at increasing luminosity and higher pileup, up to the foreseen upgrade luminosity of $2 \times 10^{33} \mathrm{~cm}^{-2} \mathrm{~s}^{-1}$, five time greater the current one. Figure 1 shows the signal to background ratio (S/B), extracted from the invariant mass distribution of $B^{ \pm} \rightarrow J / \psi(\mu \mu) K^{ \pm}$candidates, as a function of the number of primary vertices (PVs) [2]. The S/B is independent of the pileup. i.e. the number of PVs, up to 6, due to the separation between PVs, on the order of centimetres, while the resolutions of primary and secondary vertices are about $60 \mu \mathrm{m}$ and $200 \mu \mathrm{m}$ respectively.

These numbers demonstrate the performance of $\mathrm{LHCb}$ and they also introduce the concepts for the LHCb upgrade. With its upgrade LHCb aims at reaching experimental sensitivities comparable to the theoretical uncertainties. Experience with the detector operation and the analysis of the data from Run 1 shows that systematics errors can be managed and they won't spoil the final precisions. Table 1 shows the expected statistical precisions achievable in 2018 (cumulating the current 3.2 $\mathrm{fb}^{-1}$ from Run 1 and the expectation of another $5-6 \mathrm{fb}^{-1}$ from Run 2) and with the upgraded detector, for a number of representative physics modes, to be compared with the current theoretical uncertainties [3].

\section{Trigger and detector upgrade}

The LHCb upgrade is scheduled for 2018 and consists of a complete redesign of the readout and the trigger systems. Figure 2 shows how the trigger efficiencies vary as a function of the instantaneous luminosity for some reference channels. While decay modes with muons would 


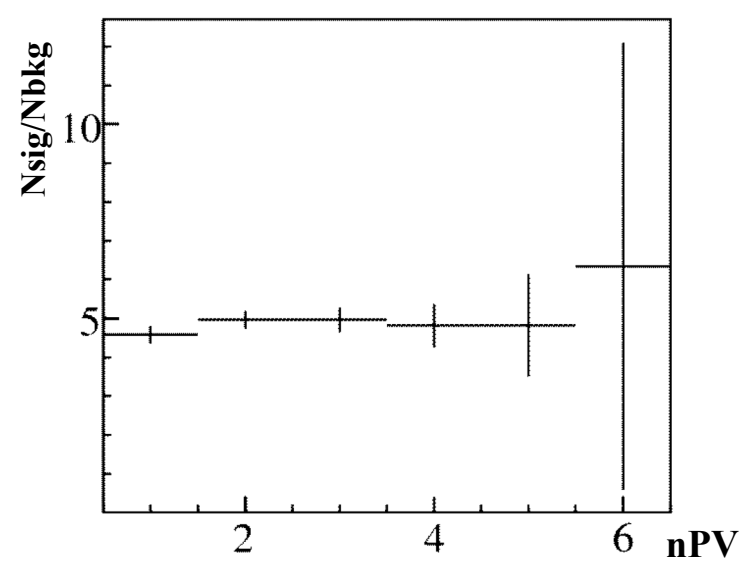

Figure 1: Signal-to-background ratio for the invariant mass distribution of $B^{ \pm} \rightarrow J / \psi(\mu \mu) K^{ \pm}$as a function of the number of primary vertices reconstructed in the event.

be handled efficiently with the present detector also at higher luminosities, the hadronic modes saturate, due to the first level trigger bandwidth limitation. An efficient trigger requires a prompt event reconstruction, based on the the whole detector information. It can be achieved by removing the hardware trigger bottleneck and reading out all the sub-detectors at $40 \mathrm{MHz}$, performing solely a software triggering on a dedicated CPU farm.

The consequence of the $40 \mathrm{MHz}$ readout requirement is that all the sub-detectors readout electronics must be rebuilt. Sub-detectors upgrades are needed in order to maintain the same performance as now, while coping with significantly higher pileup and occupancy. The main detector replacement concerns the tracking system [4], involving the VErtex LOcator (VELO) and the tracking stations. They will be completely replaced. The VELO will be based on pixel technology to provide significantly higher granularity than now. The plan is to use planar silicon sensors $55 \times 55$ $\mu \mathrm{m}^{2}$, incorporating $256 \times 256$ pixels. In order to improve the impact parameter resolution the sensor distance to the beam will be reduced by $2 \mathrm{~mm}$ (the inner radius of the RF foil will be about $3.5 \mathrm{~mm}$ from the beam instead of $5.5 \mathrm{~mm}$, with reduced foil thickness). The TT tracker will be rebuilt using silicon strips as presently, but with higher segmentation and improved sensor overlap. It will be placed closer to the beam pipe to improve the small-angle coverage. The VELO-TT system shall allow a fast momentum measurement and to efficiently resolve fake tracks. The Inner Tracker (IT) of the other stations will be entirely replaced with large surface of scintillating fibre trackers. The fibre trackers will use $250 \mu$ m diameter fibres, read out with Silicon Photo-Multipliers (SiPMs). The expected spatial resolution is about $60-100 \mu \mathrm{m}$. The straw tube geometry of the Outer Tracker (OT) can be reduced accordingly, such to overcome problems with the high occupancy in the central region of the tracker stations. The first muon detector layer (M1) located in front of the calorimeter will be removed, as well as the scintillating pad detector (SPD) and the calorimeter pre-shower used for e $\gamma$ separation, as they will not contribute with the high occupancy [5]. This is expected to improve the resolution of the electromagnetic calorimeter and ease the calibration. The e/ $\gamma$ separation will be done in the software trigger with the full information available. The calorimeter will reduce the gain of the photomultipliers and compensate with an 
increased electronics gain. The muon stations, located after the calorimeter, will remain the same. The hybrid photo-multipliers (HPD) of the two RICHes, will be replaced with multi-anode photo multipliers. The RICH1 optics will be re-optimised. Its Aerogel radiator will be removed, since it gives too few photons to actually allow reconstructing the rings in the higher multiplicities of the upgrade.

New PCIe40 readout boards will be used for data acquisition. The PCIe40 is a generic hardware component, designed for reading out all the sub-detectors, and to provide the distribution of the timing, commands and control signals [6]. The PCIe40 is based on the PCI Express Generation 3 standard protocol to connect to the event-building PC's motherboards. A cost-effective implementation of the DAQ can be achieved by embedding the readout boards into PC servers, allowing using high-speed data-centre technology in the event-building network.

\section{Conclusions}

LHCb will undergo a major upgrade between 2018 and 2019 to allow operating the experiment at a luminosity of $2 \times 10^{33} \mathrm{~cm}^{-2} \mathrm{~s}^{-1}$. The detector upgrade consists of a re-build of the sub-detectors and a complete redesign of the readout system, performed in order to read out the full detector at the bunch crossing rate of $40 \mathrm{MHz}$. In order to maximise trigger efficiencies and to minimise systematic uncertainties in the selection of the interesting flavour decays, events will be processed at the full speed by a versatile software trigger only. The upgraded readout and trigger system, as well as the detector optimisation, will allow the LHCb physics program and running conditions to adapt to any signature, which may come out as the consequence of a changing physics scene. The plan is to collect a dataset of at least $50 \mathrm{fb}^{-1}$ in less than ten years. It will allow LHCb to reach unprecedented precision in flavour physics.

\section{References}

[1] LHCb collaboration, A. A. Alves Jr. et al., The LHCb detector at the LHC, JINST 3, 2008, S08005.

[2] LHCb Collaboration, Letter of Intent for the LHCb Upgrade, CERN-LHCC-2011-001

[3] LHCb Collaboration, Framework TDR for the LHCb upgrade, CERN-LHCC-2012-007

[4] LHCb Collaboration, LHCb Tracker Upgrade Technical Design Report, CERN-LHCC-2014-001

[5] LHCb Collaboration, LHCb PID Upgrade Technical Design Report, CERN-LHCC-2013-022

[6] LHCb Collaboration, LHCb Trigger and Online Upgrade Technical Design Report, CERN-LHCC-2014-016 


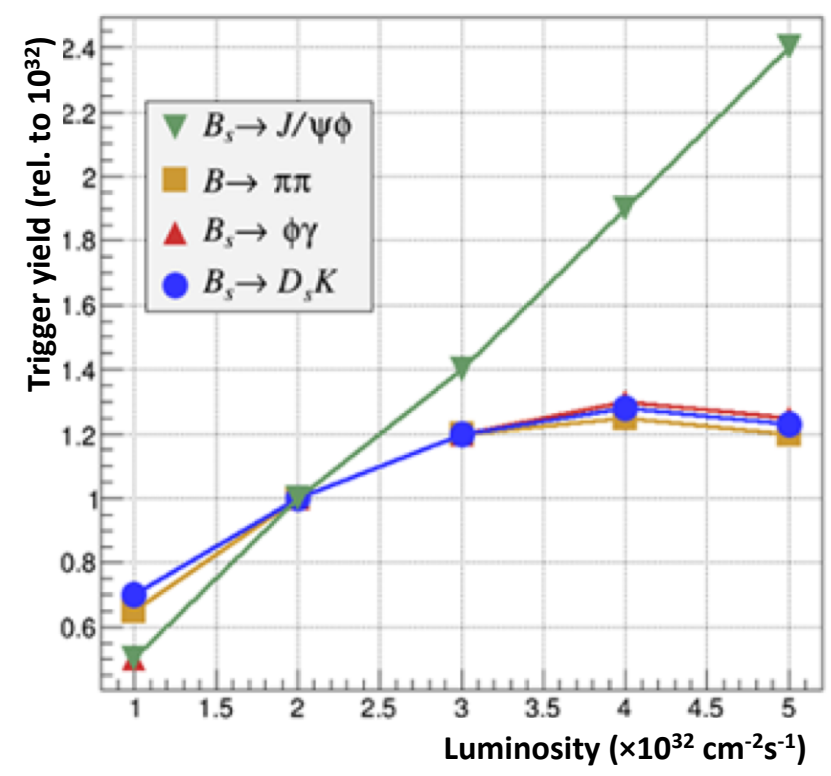

Figure 2: The plot shows the yield as a function of luminosity for different decay modes.

Table 1: Expected statistical sensitivities after LHC Run 2 and after $50 \mathrm{fb}^{-1}$ with the LHCb Upgrade as compared to current theoretical uncertainties for a list of key observables [3].

\begin{tabular}{cccccc}
\hline \hline Type & Observable & $\begin{array}{c}\text { Current } \\
\text { precision }\end{array}$ & $\begin{array}{c}\text { LHCb } \\
2018\end{array}$ & $\begin{array}{c}\text { Upgrade } \\
\left(50 \mathrm{fb}^{-1}\right)\end{array}$ & $\begin{array}{c}\text { Theory } \\
\text { uncertainty }\end{array}$ \\
\hline$B_{s}^{0}$ mixing & $2 \beta_{s}\left(B_{s}^{0} \rightarrow J / \psi \phi\right)$ & 0.10 & 0.025 & 0.008 & $\sim 0.003$ \\
& $2 \beta_{s}\left(B_{s}^{0} \rightarrow J / \psi f_{0}(980)\right)$ & 0.17 & 0.045 & 0.014 & $\sim 0.01$ \\
& $A_{\mathrm{fs}}\left(B_{s}^{0}\right)$ & $6.4 \times 10^{-3}$ & $0.6 \times 10^{-3}$ & $0.2 \times 10^{-3}$ & $0.03 \times 10^{-3}$ \\
\hline Gluonic & $2 \beta_{s}^{\text {eff }}\left(B_{s}^{0} \rightarrow \phi \phi\right)$ & - & 0.17 & 0.03 & 0.02 \\
penguin & $2 \beta_{s}^{\text {eff }}\left(B_{s}^{0} \rightarrow K^{* 0} \bar{K}^{* 0}\right)$ & - & 0.13 & 0.02 & $<0.02$ \\
& $2 \beta^{\text {eff }}\left(B^{0} \rightarrow \phi K_{S}^{0}\right)$ & 0.17 & 0.30 & 0.05 & 0.02 \\
\hline Right-handed & $2 \beta_{s}^{\text {eff }}\left(B_{s}^{0} \rightarrow \phi \gamma\right)$ & - & 0.09 & 0.02 & $<0.01$ \\
currents & $\tau^{\text {eff }}\left(B_{s}^{0} \rightarrow \phi \gamma\right) / \tau_{B_{s}^{0}}$ & - & $5 \%$ & $1 \%$ & $0.2 \%$ \\
\hline Electroweak & $S_{3}\left(B^{0} \rightarrow K^{* 0} \mu^{+} \mu^{-} ; 1<q^{2}<6 \mathrm{GeV}^{2} / c^{4}\right)$ & 0.08 & 0.025 & 0.008 & 0.02 \\
penguin & $s_{0} A_{\mathrm{FB}}\left(B^{0} \rightarrow K^{* 0} \mu^{+} \mu^{-}\right)$ & $25 \%$ & $6 \%$ & $2 \%$ & $7 \%$ \\
& $A_{\mathrm{I}}\left(K \mu^{+} \mu^{-} ; 1<q^{2}<6 \mathrm{GeV}^{2} / c^{4}\right)$ & 0.25 & 0.08 & 0.025 & $\sim 0.02$ \\
& $\mathcal{B}\left(B^{+} \rightarrow \pi^{+} \mu^{+} \mu^{-}\right) / \mathcal{B}\left(B^{+} \rightarrow K^{+} \mu^{+} \mu^{-}\right)$ & $25 \%$ & $8 \%$ & $2.5 \%$ & $\sim 10 \%$ \\
\hline Higgs & $\mathcal{B}\left(B_{s}^{0} \rightarrow \mu^{+} \mu^{-}\right)$ & $1.5 \times 10^{-9}$ & $0.5 \times 10^{-9}$ & $0.15 \times 10^{-9}$ & $0.3 \times 10^{-9}$ \\
penguin & $\mathcal{B}\left(B^{0} \rightarrow \mu^{+} \mu^{-}\right) / \mathcal{B}\left(B_{s}^{0} \rightarrow \mu^{+} \mu^{-}\right)$ & - & $\sim 100 \%$ & $\sim 35 \%$ & $\sim 5 \%$ \\
\hline Unitarity & $\left.\gamma\left(B \rightarrow D^{*}\right) K^{(*)}\right)$ & $\sim 10-12^{\circ}$ & $4^{\circ}$ & $0.9^{\circ}$ & negligible \\
triangle & $\gamma\left(B_{s}^{0} \rightarrow D_{s} K\right)$ & - & $11^{\circ}$ & $2.0^{\circ}$ & negligible \\
angles & $\beta\left(B^{0} \rightarrow J / \psi K_{S}^{0}\right)$ & $0.8^{\circ}$ & $0.6^{\circ}$ & $0.2^{\circ}$ & negligible \\
\hline Charm & $A_{\Gamma}$ & $2.3 \times 10^{-3}$ & $0.40 \times 10^{-3}$ & $0.07 \times 10^{-3}$ & - \\
$C P$ violation & $\Delta A_{C P}$ & $2.1 \times 10^{-3}$ & $0.65 \times 10^{-3}$ & $0.12 \times 10^{-3}$ & - \\
\hline \hline
\end{tabular}

